\title{
Consumo de basuco en mujeres transgénero, de tres ciudades de Colombia
}

\section{Basuco consumption in transgender women across three cities in Colombia}

\author{
Sara Milena Ramos Jaraba*, Dedsy Yajaira Berbesí Fernández*, Elizabeth Trejos-Castillo**. \\ * Facultad de Enfermería, Universidad CES, Medellín, Colombia. \\ ** Human Development \& Family Sciences, Texas Tech University, Lubbock, TX.
}

\section{Resumen}

Este artículo tuvo como objetivo describir los factores asociados al consumo de basuco en mujeres transgénero de tres ciudades de Colombia. Se realizó un estudio descriptivo transversal, a través del muestreo dirigido por los entrevistados (MDE) y en el cual participaron 688 mujeres transgénero de Bogotá, Medellín y Santiago de Cali. Se realizaron análisis descriptivos, asociaciones bivariadas y una regresión de tipo binomial. La prevalencia del consumo de basuco fue de $11 \%$, y los factores asociados a su consumo entre las MT fueron, ser de estrato socioeconómico bajo, vivir sola, percibirse vulnerable al VIH, haber tenido una ITS en el último año y consumir marihuana. El consumo de sustancias en las MT en Colombia es elevado al compararlo con población general, se evidencia que el consumo de basuco se enmarca en el contexto de vulnerabilidad social. Es necesario incentivar la creación de políticas y programas con un abordaje integral en salud, que tengan como uno de los ejes de acción la prevención del consumo de sustancias psicoactivas entre las mujeres transgénero, teniendo en cuenta sus particularidades y características sociodemográficas y económicas.

Palabras clave: Mujeres transgénero; basuco; consumo de sustancias psicoactivas; Colombia.

\begin{abstract}
This article aims to describe the factors associated with basuco consumption in transgender women (TW) in three cities in Colombia, South America. A cross-sectional descriptive study was carried out using Respondent-Driven Sampling (RDS) and included 688 transgender women participants from Bogotá, Medellín and Santiago de Cali. Descriptive analyses, bivariate associations, and a binomial regression were performed. The prevalence of basuco consumption among participants was $11 \%$; factors associated with basuco consumption among TW included having a low socioeconomic level, living alone, feeling vulnerable to HIV, having had an STI in the last year, and consuming marijuana. Substance consumption in TW in Colombia is high when compared to the general population, and the literature shows that basuco consumption is intensified within a context of social vulnerability. Encouraging the development of policies and programs with a comprehensive approach to health and substance use prevention particularly among vulnerable TW considering their unique sociodemographic and economic characteristics, is warranted. Key words: Transgender women; basuco; substance use; Colombia.
\end{abstract}


L as mujeres transgénero (MT) constituyen un grupo de investigación prioritario en salud pública, sufren inequidades de género, estigma y discriminación, exclusión social, patologización de su identidad de género, gozan de pocas oportunidades y garantías educativas y laborales, asimismo condiciones de salud precarias, pues sus necesidades particulares han sido invisibilizadas (Radi y Sardá-Chandiraman, 2016; Scher, 2016). Algunos informes reportan que en América Latina (AL) las MT, toleran una carga desproporcionada de enfermedad, invalidez, y riesgos que les impiden el goce efectivo de los derechos en salud, a la vez un aumento inmensurable de muertes violentas por prejuicio, maltrato policial, persecución y una esperanza de vida baja. Además, se estima que más del $50 \%$ de ellas se desempeñan como vendedoras ambulantes, o trabajadoras sexuales, tienen bajo nivel educativo, no tienen acceso a vivienda y una cuarta parte no accede a los servicios de salud (Comisión Interamericana de Derechos Humanos, 2015; Organización Panamericana de la Salud, 2011; Sandoval-Rebollo, Domínguez-Cornejo y Rosales-Galarza, 2019; SInViolencia LGBT, 2019).

Frente a la precarización en salud, las MT enfrentan múltiples problemas, se encuentran, por ejemplo, las consecuencias derivadas de las transformaciones corporales inseguras, problemas de salud reproductiva y a las Infecciones de Transmisión Sexual (ITS), especialmente el VIH. Como resultado del estigma y la transfobia se ha comprobado que presentan problemas de salud mental, como el estrés, la depresión, ansiedad, ideación suicida y el desorden de estrés postraumático (Bazargan y Galvan, 2012; Organización Panamericana de la Salud, 2011; Yarns, Abrams, Meeks y Swell, 2016). Además, las MT se encuentran entre los grupos más vulnerables al consumo de sustancias psicoactivas (SPA).

En Colombia los estudios de la última década señalan un aumento crítico en el consumo de sustancias psicoactivas (SPA), especialmente entre la población joven, y se reportan como las comúnmente utilizadas el tabaco, el alcohol, la marihuana, la cocaína y sus derivados, tales como la pasta básica de coca, llamada en algunos países latinoamericanos, como basuco (Ministerio de Salud y Protección Social, 2015). El consumo de basuco, pitillo o Kete como es llamada la pasta de coca en diferentes países de América Latina, denota un problema de desigualdades sociales y de los grupos menos favorecidos, por lo cual no ha sido objeto de investigaciones, a diferencia de la cocaína; en este sentido faltan datos cualitativos y cuantitativos realmente fiables que ayuden a explicar su consumo. Las impurezas del producto y las sustancias agregadas, hacen que tenga un menor costo en el mercado en comparación con el clorhidrato de cocaína (Téllez-Mosquera y Cote-Menéndez, 2005). Se sabe que el basuco es mucho más perjudicial que la cocaína por sus impurezas, y las sustancias que se usan para su preparación, estas hacen que no sea administrable por vía intravenosa o intramuscular, y su uso se de en forma de cigarrillos, pipas y otras vías de empleo pulmonar (Larrea-Torrelio, 2007).

Al basuco también se le conoce en Norteamérica como "Crack cocaine", "Rock" y "Freebase", pero denota algunas diferencias. El crack surge de mezclar el clorhidrato de cocaína con bicarbonato de sodio y agua o amoniaco, mientras que el basuco es un extracto crudo de la hoja de coca mezclado con agua, ácido sulfúrico y querosén, gasolina o agua de batería, lo que lo convierte en un componente intermedio en la preparación del clorhidrato de cocaína (Dávila, Solórzano, Premoli de Percoco, Quiñones y Petrosino, 2001).

La amplia disponibilidad y el bajo costo del basuco en las áreas urbanas hacen muy fácil su consumo en grandes cantidades; de hecho, el precio carece de importancia si la persona está implicada en la distribución del producto. En Colombia, los centros poblados con más de un millón de habitantes reportan mayor consumo, en este orden de ideas, Bogotá, Medellín con área metropolitana y Cali presentan las mayores prevalencias de uso de cocaína, por lo que hay indicios de un patrón similar de consumo de basuco (Ministerio de Salud y Protección Social, 2015). Estudios han puesto en evidencia que el perfil epidemiológico de los consumidores de basuco corresponde generalmente a personas de estratos bajos, escasa formación educativa y en su mayoría hombres (Isaza, Suárez, Henao y González, 2010; Téllez-Mosquera et al., 2005). Por otro lado, son conocidos los efectos del consumo de basuco y cocaína, pues son drogas psicotrópicas capaces de estimular o acelerar la actividad del sistema nervioso central, y su acción puede repercutir sobre los diversos sistemas del organismo. El consumo de basuco puede ser predictivo a generar un policonsumo, aumentar el número de dosis en usuarios de drogas inyectables, y la probabilidad de adquirir una enfermedad de transmisión sexual y de seropositividad para el virus de la inmunodeficiencia humana y de la hepatitis C (Berbesi-Fernández, Segura-Cardona, Montoya-Vélez y Hernández-Rendón, 2016).

En las MT no hay mucha evidencia de consumo específico de basuco, algunos informes sugieren un consumo frecuente de alcohol y otras sustancias, en especial entre las personas trans que ejercen el trabajo sexual o que se encuentran en situación de calle (Ecker, Aubry y Sylvestre, 2019; Scheim, Bauer y Shokoohi, 2017). Las sustancias de mayor consumo son: el alcohol, la marihuana, la cocaína y sus derivados ( $R e-$ back y Fletcher, 2014). Además, las prevalencias de consumo de MT son mayores a las de población general, y hasta diez veces mayores que la de hombres trans (HT) (Gómez-Gil et al., 2019). Este consumo puede estar asociado a la búsqueda de situaciones que permitan soportar las realidades de un entorno generalmente difícil, y con el fin de reducir los efectos del alcohol (Gómez-Gil et al., 2019; Yarns et al., 2016). Entre los factores asociados, se ha encontrado como la experiencia previa de abuso sexual, aumentó casi en tres veces la posibilidad de consumir cocaína en MT de Repúbli- 
ca Dominicana (Budhwani et al., 2017). En MT de Canadá el consumo aumentaba entre quienes tuvieron experiencias de transfobia, eran habitantes de calle, y trabajadoras sexuales (Scheim et al., 2017). Como consecuencia el abuso de SPA puede reducir la capacidad de negociar los términos de un encuentro sexual, lo que aumenta la vulnerabilidad y el riesgo de infección por VIH, a la violencia sexual, entre otras (Santos et al., 2014; Scheim et al, 2017).

A pesar del aumento sistemático de consumo de SPA que señalan los estudios disponibles en Colombia y a los avances que ha tenido el país en el desarrollo de estudios epidemiológicos en población escolar y general, las de producción, el tráfico y sus consecuencias, poco se ha investigado sobre el consumo de basuco. Esta carencia de atención puede estar ligada a que su consumo generalmente se ha atribuido a población habitante de calle, sin embargo, estos patrones pueden estar cambiando y otros grupos vulnerables, como son las MT han presentado un consumo incipiente de esta substancia.

Dada la carencia de información sobre el consumo de basuco en MT latinoamericanas, unido a la limitada información sobre hábitos de consumo o abuso de alcohol, tabaco y drogas ilícitas en la población trans que ha ido creciendo en los últimos anos, este artículo tiene como objetivo caracterizar los factores asociados al consumo de basuco en MT en Colombia.

\section{Método}

\section{Tipo y diseño general del estudio}

Se realizó un estudio descriptivo transversal, usando la base de datos del estudio nacional titulado: "Vulnerabilidad al VIH y prevalencia de VIH en mujeres transgénero en tres ciudades de Colombia: Bogotá, Medellín y Santiago de Cali, 2019" entre mayo y septiembre de 2019 (Berbesi et al., 2019). Las ciudades priorizadas para este estudio, son las tres con mayor estimación de mujeres trans en Colombia y además presentan las mayores prevalencias de VIH en población clave: Hombres que tienen sexo con hombres y mujeres transgénero (Ministerio de Justicia y del Derecho y Ministerio de Salud y Protección Social, 2014).

\section{Procedimientos y participantes}

Para el muestreo se utilizó muestreo dirigido por los entrevistados (MDE; Respondent Driving Sampling RDS), esta metodología es ideal para alcanzar representatividad de la muestra en aquellos grupos en que desconocemos el marco muestral o que son llamado "poblaciones ocultas", como lo son las mujeres transgénero. El reclutamiento es similar a la bola de nieve o al de referenciamiento en cadena, pues mediante la recopilación de los datos del tamaño de la red social y limitando la entrega de cupones, los resultados pueden ajustarse y representar las redes de la población muestreada (Carballo-Diéguez et al., 2011).
Para el proceso de reclutamiento y teniendo en cuenta la teoría del RDS, se convocaron inicialmente nueve semillas (3 en cada ciudad), el reclutamiento finalizó con la inclusión de 20 semillas en las tres ciudades para lograr el tamaño de la muestra requerida. Estas fueron identificadas a través de organizaciones sociales, actores clave de la población y actores institucionales. Se seleccionaron como semillas a aquellas personas que manifestaron poder convocar a un numeroso grupo de mujeres trans, que tuvieran liderazgo, visibilidad, reconocimiento y fueran diversas en cuando a sus características sociodemográficas, y que cumplieran los criterios mínimos de elegibilidad: asignación de sexo masculino al nacer, identificarse con el género femenino, ser mayor de edad y residir en las ciudades de Bogotá, Medellín (área metropolitana), y Cali (área metropolitana) y tener ciudadanía colombiana.

Todas las participantes, incluyendo las semillas, recibieron tres cupones para reclutar a otras participantes elegibles de sus redes sociales. Este sistema de reclutamiento en cadena se continuó hasta alcanzar el total de 668 participantes (sin semillas). Siguiendo la teoría del RDS a cada persona se le dio un incentivo por su participación en el estudio (bono de supermercado por valor de 40.000 COP, aproximadamente 12 USD) y otro secundario vinculado al reclutamiento exitoso de tres nuevos participantes (dinero en efectivo, 30.000 COP, aproximadamente 9 USD). El tamaño de la muestra se alcanzó en un plazo de dieciocho semanas.

\section{Instrumento $\boldsymbol{y}$ variables}

Se aplicó una encuesta adaptada de acuerdo a las directrices para encuestas del comportamiento repetidas en poblaciones en riesgo al VIH, (Family Health International, 2000) la cual fue ajustada en Colombia por un grupo de expertos de la entidad financiadora. Esta encuesta estaba dividida en 12 secciones: características sociales y demográficas, salud y acceso a servicios, transformaciones del cuerpo, historia sexual, infecciones de transmisión sexual, ejercicio del trabajo sexual, condón y lubricante, conocimientos sobre VIH/SIDA, exposición, intervención y prueba de VIH, consumo de sustancias psicoactivas, redes sociales y estigma/discriminación, además se realizaron pruebas rápidas para VIH y aquellas que tuvieran un resultado reactivo, se confirmaron a través de una prueba Elisa en papel filtro. Como variable de desenlace se utilizó consumo de basuco a través de la pregunta ¿En los últimos 6 meses, ha fumado o inhalado pipa, crack o basuco?, siendo las respuestas sí o no. Las variables de control del estudio fueron: edad, nivel educativo (ninguno/preescolar/primaria, secundaria, técnico o tecnológico y universidad/posgrado), ocupación (empleada/independiente, estilista/modista, ninguna/estudiante y prostitución/show/webcam), estado civil (soltera y casada/unión libre/viuda), estrato socioeconómico (utilizado en Colombia como la clasificación de los inmuebles residenciales que deben recibir servicios públicos, a menor 
estrato menor capacidad económica), ingresos (de acuerdo a los salarios mínimos legales mensuales vigentes en Colombia -SMMLV- a 2019, que eran aproximadamente 925.000 COP o 289 USD), vive sola (si o no), apoyo social (si o no), vulnerabilidad al VIH (bajo, media-alta), infecciones de transmisión sexual en el último año (si o no), diagnóstico de VIH (positivo o negativo), discriminación en el último año (si o no), dinero a cambio de sexo alguna vez en la vida (si o no), consumo de cocaína (si o no), marihuana (si o no) y alcohol (si o no), en los últimos 6 meses, relaciones forzosas en el último año (si o no), parejas ocasionales en 6 meses (si o no) y molestias/problemas de salud en el último año (si o no).

Las encuestas fueron aplicadas de manera electrónica, por personal entrenado para tal fin. Se realizaron en una sede ubicada en un lugar accesible para la población en cada ciudad.

Este estudio fue aprobado por el comité de ética de la Universidad CES, en sesión del 19 de febrero de 2019. Se siguieron los requerimientos de las Normas Científicas, Técnicas y Administrativas para la Investigación en Salud, según la Resolución 008430 del 4 de octubre de 1993 del Ministerio de Salud de Colombia, clasificada como investigación con riesgo mínimo. Todas las participantes firmaron un consentimiento informado donde aceptaron voluntariamente hacer parte del estudio.

\section{Análisis}

Se realizaron análisis bivariados utilizando chi cuadrado, para lo cual se excluyeron los registros de las MT que no tuvieran dato si habían consumido o no basuco, resultando en un total de 618 observaciones; luego se construyeron dos modelos de regresión logística (sin ajustar y ajustado) con el fin de explorar los factores asociados al consumo de basuco. En los modelos de regresión logística se incluyó la variable de desenlace (basuco) con cada una de las variables del estudio a través del método Introducir. En el modelo multivariado se ingresaron las variables significativas

Tabla 1. Características sociodemográficas de acuerdo con el consumo de basuco en MT.

\begin{tabular}{|c|c|c|c|c|}
\hline \multirow{3}{*}{ Variables } & \multirow[b]{3}{*}{$(n \%)$} & \multicolumn{2}{|c|}{ Basuco } & \multirow{3}{*}{$p^{\star}$} \\
\hline & & Sí & No & \\
\hline & & $68(11,1 \%)$ & $549(88,9 \%)$ & \\
\hline \multicolumn{5}{|l|}{ Edad } \\
\hline 18 a 24 años & $187(27,2)$ & $14(7,5)$ & $173(92,5)$ & \multirow{4}{*}{0,048} \\
\hline 25 a 34 años & $249(36,2)$ & $29(11,6)$ & $220(88,4)$ & \\
\hline 35 a 44 años & $106(15,4)$ & $19(17,9)$ & $87(82,1)$ & \\
\hline 45 años y más & $146(21,2)$ & $14(9,6)$ & $132(90,4)$ & \\
\hline \multicolumn{5}{|l|}{ Ocupación } \\
\hline Empleada/independiente & $104(17,6)$ & $17(16,3)$ & $87(83,7)$ & \multirow{4}{*}{0,052} \\
\hline Estilista/peluquera/modista & $139(23,5)$ & $8(5,8)$ & $131(94,2)$ & \\
\hline Ninguna/estudiante & $71(12,0)$ & $15(21,1)$ & $56(78,9)$ & \\
\hline Prostitución/ Show/webcam & $277(46,9)$ & $28(10,1)$ & $249(89,9)$ & \\
\hline \multicolumn{5}{|l|}{ Estrato } \\
\hline Estrato 1 & $158(26,5)$ & $34(21,5)$ & $124(78,5)$ & \multirow{3}{*}{0,001} \\
\hline Estrato 2 & $200(33,6)$ & $14(7,0)$ & $186(93,0)$ & \\
\hline Estrato 3 y más & $238(39,9)$ & $20(8,4)$ & $218(91,6)$ & \\
\hline \multicolumn{5}{|l|}{ Estado civil } \\
\hline Soltera & $483(79,1)$ & $55(11,4)$ & $428(88,6)$ & \multirow{2}{*}{0,416} \\
\hline Casada/unión libre/viuda & $128(20,9)$ & $13(10,2)$ & $115(89,8)$ & \\
\hline \multicolumn{5}{|l|}{ Nivel educativo } \\
\hline Ninguno/preescolar/primaria & $86(13,9)$ & $13(15,1)$ & $73(84,9)$ & \multirow{3}{*}{0,087} \\
\hline Secundaria & $424(68,6)$ & $49(11,6)$ & $375(88,4)$ & \\
\hline Técnico/tecnológico & $108(17,5)$ & $6(5,6)$ & $102(94,4)$ & \\
\hline \multicolumn{5}{|l|}{ Ingresos } \\
\hline Sin ingresos & $81(13,2)$ & $15(18,5)$ & $66(81,5)$ & \multirow{4}{*}{0,013} \\
\hline Salario mínimo o menos & $320(52,2)$ & $41(12,8)$ & $279(87,2)$ & \\
\hline Entre uno y dos salarios mínimos & $127(20,7)$ & $7(5,5)$ & $120(94,5)$ & \\
\hline Más de dos salarios mínimos & $85(13,9)$ & $6(7,1)$ & $79(92,9)$ & \\
\hline \multicolumn{5}{|l|}{ Vive solo } \\
\hline Sí & $195(31,5)$ & $80(41,0)$ & $115(59,0)$ & \multirow[b]{2}{*}{0,028} \\
\hline No & $425(68,5)$ & $139(32,7)$ & $286(67,3)$ & \\
\hline \multicolumn{5}{|l|}{ Apoyo social } \\
\hline Sí & $367(60,0)$ & $30(8,2)$ & $337(91,8)$ & \multirow{2}{*}{0,002} \\
\hline No & $425(68,5)$ & $139(32,7)$ & $286(67,3)$ & \\
\hline
\end{tabular}

*Nota: Valores $p<0,05$ en negrita. 
$(p<0,05)$ en el análisis bivariado. Los datos se analizaron en RDSAT y se ponderaron en el SPSS $®$, versión 21, con licencia institucional.

\section{Resultados}

Los resultados denotan que las MT participantes de la investigación son en su mayoría jóvenes, con una edad promedio de 32,9 años (DE = 12,7), el 46,9\% tienen como ocupación el trabajo sexual/webcam y shows, seguidas del 23,5\% que son estilistas/peluqueras o modistas, y de acuerdo al estrato socioeconómico se encontró que el 39,9\% son de estrato tres o más, $33,6 \%$ son de estrato dos y $26,5 \%$ son de estrato uno. Predomina el estado civil soltera $(79,1 \%)$, el porcentaje restante se encontraban casadas, en unión libre o viudas; según el nivel educativo el 86,1\% terminó el bachillerato o tiene estudios superiores. Más de la mitad de las MT recibe mensualmente un salario mínimo o menos de este, el 68,5\% vive con alguien, el 35,6\% tiene a cargo personas dependientes y $60,0 \%$ cuenta con apoyo o ayuda de un familiar o amigo en caso de necesitarlo (Tabla 1).

Con relación a otras características, se encontró que más del $62,7 \%$ de las MT se perciben vulnerables al VIH, $12,8 \%$ ha tenido alguna ITS en el último año, el 82,5\% ha sufrido algún tipo de discriminación, $4,1 \%$ fue obligada a tener relaciones sexuales en el último año, $12,1 \%$ de las encuestadas dijo no usar condón en las relaciones sexuales, el 49,3\% ha tenido parejas ocasionales en el último año y $39,1 \%$ ha tenido molestias de salud en el último año (Tabla 2).

Tabla 2. Características comportamentales de acuerdo con el consumo de basuco en MT.

\begin{tabular}{|c|c|c|c|c|}
\hline \multirow{3}{*}{ Variables } & \multirow[b]{3}{*}{ (n \%) } & \multicolumn{2}{|c|}{ Basuco } & \multirow{3}{*}{$p^{*}$} \\
\hline & & Sí & No & \\
\hline & & $76(11)$ & $612(89)$ & \\
\hline \multicolumn{5}{|c|}{ Vulnerabilidad al VIH } \\
\hline Bajo & $200(37,3)$ & $11(5,5)$ & $189(94,5)$ & \multirow[t]{2}{*}{0,002} \\
\hline Medio-alto & $336(62,7)$ & $45(13,4)$ & $291(86,6)$ & \\
\hline \multicolumn{5}{|l|}{ VIH } \\
\hline Positivo & $167(27,3)$ & $23(13,8)$ & $144(86,2)$ & \multirow[t]{2}{*}{0,130} \\
\hline Negativo & $444(72,7)$ & $45(10,1)$ & $399(89,9)$ & \\
\hline \multicolumn{5}{|l|}{ ITS } \\
\hline Sí & $78(12,8)$ & $20(25,9)$ & $58(74,4)$ & \multirow[t]{2}{*}{0,000} \\
\hline No & $533(87,2)$ & $48(9,0)$ & $485(91,0)$ & \\
\hline \multicolumn{5}{|l|}{ Cocaína } \\
\hline Sí & $187(30,6)$ & $29(15,5)$ & $158(84,5)$ & \multirow[t]{2}{*}{0,018} \\
\hline No & $424(69,4)$ & $39(9,2)$ & $385(90,8)$ & \\
\hline \multicolumn{5}{|l|}{ Marihuana } \\
\hline Sí & $316(51,7)$ & $55(17,4)$ & $261(82,6)$ & \multirow[t]{2}{*}{0,000} \\
\hline No & $295(48,3)$ & $13(4,4)$ & $282(95,6)$ & \\
\hline \multicolumn{5}{|l|}{ Alcohol } \\
\hline Sí & $431(70,7)$ & $42(9,7)$ & $389(90,3)$ & \multirow[t]{2}{*}{0,061} \\
\hline No & $179(23,3)$ & $26(14,5)$ & $153(85,5)$ & \\
\hline \multicolumn{5}{|c|}{ Discriminación } \\
\hline Sí & $107(17,5)$ & $15(14,0)$ & $92(86,0)$ & \multirow{2}{*}{0,205} \\
\hline No & $504(82,5)$ & $54(10,7)$ & $450(89,3)$ & \\
\hline \multicolumn{5}{|c|}{ Relaciones sexuales forzosas en el último año } \\
\hline Sí & $25(4,1)$ & $6(24,0)$ & $19(76,0)$ & \multirow[t]{2}{*}{0,049} \\
\hline No & $586(95,9)$ & $62(10,6)$ & $524(89,4)$ & \\
\hline \multicolumn{5}{|c|}{ Usa preservativo } \\
\hline Sí & $537(87,9)$ & $57(10,6)$ & $480(89,4)$ & \multirow[t]{2}{*}{0,184} \\
\hline No & $74(12,1)$ & $11(14,9)$ & $63(85,1)$ & \\
\hline \multicolumn{5}{|c|}{ Recibió dinero a cambio de sexo por lo menos una vez en la vida } \\
\hline Sí & $491(80,5)$ & $62(12,6)$ & $429(87,4)$ & \multirow[t]{2}{*}{0,010} \\
\hline No & $119(19,5)$ & $6(5,0)$ & $113(95,0)$ & \\
\hline \multicolumn{5}{|c|}{ Parejas ocasionales en 6 meses } \\
\hline Sí & $301(49,3)$ & $28(9,3)$ & $273(90,7)$ & \multirow[b]{2}{*}{0,099} \\
\hline No & $310(50,7)$ & $40(12,9)$ & $270(87,1)$ & \\
\hline \multicolumn{5}{|c|}{ Molestias o problemas de salud } \\
\hline Sí & $239(39,1)$ & $43(18,0)$ & $196(82,0)$ & 0,000 \\
\hline No & $373(60,9)$ & $26(7,0)$ & $347(93,0)$ & \\
\hline
\end{tabular}

*Nota: Valores $p<0,05$ en negrita. 
Con relación al consumo de SPA, 30,6\% reportó haber consumido cocaína, $51,7 \%$ marihuana, $70,7 \%$ alcohol y $11 \%$ basuco en los 6 meses previos a la encuesta, mostrando diferencias importantes por ciudad. Bogotá es la ciudad con mayor prevalencia de consumo de basuco $(17,6 \%)$, Medellín reporta la mayor prevalencia de consumo de marihuana $(61,6 \%)$ y Cali reporta el mayor consumo de Cocaína $(37,7 \%)$ (Figura 1).
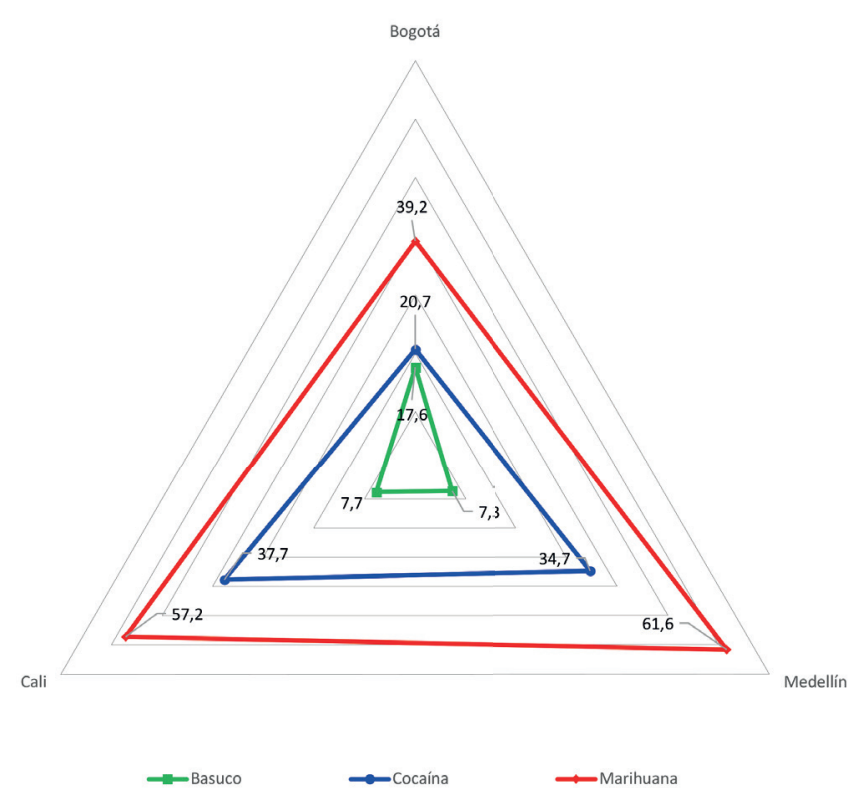

Figura 1. Prevalencias de consumo de sustancias psicoactivas por ciudad en mujeres trans en Colombia.

Al realizar el análisis entre el consumo de basuco y las variables de interés, se encontró una asociación estadísticamente significativa entre el consumo de basuco y tener entre 25 a 44 años, ser de estrato socioeconómico uno, no tener apoyo social, vivir sola, percibirse vulnerable al VIH, haber tenido una ITS, consumir cocaína, marihuana, haber sido forzada a tener relaciones sexuales en el último año, haber recibido dinero a cambio de sexo alguna vez en la vida, y haber tenido problemas de salud en el último año.

En el modelo de regresión logística, se mantuvieron significativas las mismas variables a excepción de la edad. Al comparar las MT que consumen basuco frente a las no consumidoras de esta sustancia, se encontró que ser de estrato uno aumenta en más de tres veces la posibilidad de consumo (OR 3,65; IC-1,291-5,452), así mismo vivir sola en comparación a las que viven acompañadas (OR 2,75; IC 95\%-1,477-5,114).

Las MT que se perciben vulnerables al VIH, tienen 2,3 veces la probabilidad de consumir, de igual manera aumenta entre las que tuvieron una ITS en el último año en 2,9 veces (OR 2,97; IC-1,41-6,25). Se encontró que el uso de marihuana aumenta 3,4 veces la probabilidad del consumo de basuco y que entre las MT que reportaron un problema de salud también incrementa esta posibilidad de consumo, con respecto a las que no reportan problemas (OR 2,37; IC1,477-5,714) (Tabla 3).

Tabla 3. Factores asociados al consumo de basuco en MT.

\begin{tabular}{|c|c|c|}
\hline \multirow{2}{*}{ Variables } & \multirow{2}{*}{$\begin{array}{c}\text { Modelo sin ajustar } \\
\text { OR (IC 95\%) }\end{array}$} & \multirow{2}{*}{$\begin{array}{c}\text { Modelo Ajustado } \\
\text { ORA (IC 95\%) }\end{array}$} \\
\hline & & \\
\hline \multicolumn{3}{|l|}{ Edad } \\
\hline 18 a 24 años & $1,17(0,514-2,260)$ & \\
\hline 25 a 34 años & $1,74(0,817-3,688)$ & \\
\hline 35 a 44 años & $1,92(0,772-4,776)$ & \\
\hline 45 años y más & 1 & \\
\hline \multicolumn{3}{|l|}{ Estrato } \\
\hline Estrato 1 & $2,93(1,620-5,310)$ & $3,65(1,291-5,452)$ \\
\hline Estrato 2 & $0,78(0,384-1,606)$ & $0,77(0,347-1,71)$ \\
\hline Estrato 3 y más & 1 & 1 \\
\hline \multicolumn{3}{|l|}{ Ingresos } \\
\hline Sin ingresos & $3,10(1,117-8,616)$ & \\
\hline Salario mínimo o menos & $2,01(0,807-5,016)$ & \\
\hline $\begin{array}{l}\text { Entre uno y dos salarios } \\
\text { mínimos }\end{array}$ & $0,85(0,276-2,64)$ & \\
\hline Más de dos salarios mínimos & 1 & \\
\hline \multicolumn{3}{|l|}{ Vive sola } \\
\hline Sí & $2,47(1,474-4,124)$ & $2,75(1,477-5,114)$ \\
\hline No & 1 & 1 \\
\hline \multicolumn{3}{|l|}{ Apoyo social } \\
\hline Sí & 1 & \\
\hline No & $2,13(1,283-3,548)$ & \\
\hline \multicolumn{3}{|l|}{ Vulnerabilidad al VIH } \\
\hline Bajo & 1 & 1 \\
\hline Medio-alto & $2,65(1,339-5,256)$ & $2,33(1,109-4,903)$ \\
\hline \multicolumn{3}{|l|}{ ITS } \\
\hline Sí & $3,56(1,981-6,382)$ & $2,97(1,414-6,252)$ \\
\hline No & 1 & 1 \\
\hline \multicolumn{3}{|l|}{ Cocaína } \\
\hline Sí & $1,80(1,080-3,018)$ & \\
\hline No & 1 & \\
\hline \multicolumn{3}{|l|}{ Marihuana } \\
\hline Sí & $4,65(2,48-8,71)$ & $3,49(1,474-4,124)$ \\
\hline No & 1 & 1 \\
\hline \multicolumn{3}{|l|}{$\begin{array}{l}\text { Relaciones sexuales forzosas } \\
\text { en el último año }\end{array}$} \\
\hline Sí & $2,72(1,049-7,068)$ & \\
\hline No & 1 & \\
\hline \multicolumn{3}{|l|}{$\begin{array}{l}\text { Recibió dinero a cambio de sexo } \\
\text { por lo menos una vez en la vida }\end{array}$} \\
\hline Sí & $2,71(1,148-6,401)$ & \\
\hline No & 1 & \\
\hline \multicolumn{3}{|l|}{ Molestias o problemas de salud } \\
\hline Sí & $2,96(1,760-4,981)$ & $2,37(1,477-5,714)$ \\
\hline No & 1 & 1 \\
\hline
\end{tabular}

\section{Discusión}

Según el rastreo bibliográfico realizado a la fecha, este estudio es el primero en determinar el consumo de basuco en MT colombianas, encontrando que las MT tienen una prevalencia de consumo de basuco alrededor del 11\% y que a pesar de que esta sustancia es comúnmente utilizada en el país, existe poca investigación sobre su uso en grupos 
vulnerables. Los hallazgos evidencian que el consumo de esta sustancia en esta población está asociado a una combinación de condiciones socioeconómicas, comportamentales y del entorno social, que complejizan su abordaje, como ha sido reportado en otras investigaciones sobre el tema (Family Health International, 2000; SIIS Centro de Documentación y Estudios, 2014).

El perfil sociodemográfico de las usuarias de basuco muestra un panorama de vulnerabilidad social, ya que predomina el consumo en las MT de estrato uno, con una edad promedio de 32 años, que culminaron estudios de secundaria, no reciben ingresos, no trabajan o son estudiantes. Resultados similares fueron encontrados en una revisión realizada por la Organización de los Estados Americanos (2014), en usuarios de pasta base de cocaína en América Latina, quienes mencionan en común: una edad media de consumo de alrededor de 30 años, de nivel de educación media, que no reciben ingresos, no trabajaban, en un gran porcentaje eran habitantes de calle y tenían alta probabilidad de tener conductas sexuales de riesgo. Además, en este estudio otros factores que influyeron en el consumo, fueron que las MT vivieran solas, no auto percibieran apoyo social, situaciones que caracterizan sus condiciones de vida, pues por el estigma y discriminación muchas MT son expulsadas de sus hogares a temprana edad y sus redes sociales son escasas, este mismo hallazgo fue encontrado en población usuaria de drogas inyectables (Berbesi-Fernández et al., 2016).

Entre los resultados se destaca un consumo problemático de SPA entre las MT, ya que se reportan altas prevalencias del uso de cocaína, marihuana y alcohol, en porcentaje mucho mayores a los observados en mujeres cisgénero e incluso en otros grupos con altas prevalencias como son los HSH (Valencia et al., 2018). Esta situación ya conocida en consumidores de basuco fue reportada también en una encuesta nacional en Uruguay en la cual se observó que prácticamente la totalidad de las personas que consumían "pasta base" eran policonsumidores, y que más de la mitad de la demanda de rehabilitación ocurría en usuarios de esta droga, dada sus características como "altamente adictiva” y por la alta vulnerabilidad de sus consumidores (Observatorio Uruguayo de Drogas, 2014).

$\mathrm{Al}$ comparar las prevalencias de consumo encontradas en este estudio con las reportadas en otros internacionales, se encontró que en MT de San Francisco (Estados Unidos) la sustancia de mayor uso fue la marihuana (29\%), un $13,4 \%$ consumían pasta de coca o "crack cocaine", y $58 \%$ tomaban alcohol (Santos et al., 2014). Por otro lado, un estudio en Houston (Estados Unidos), reportó una prevalencia de consumo de cocaína y/o pasta de cocaína del $40 \%$, altos índices de violencia de pareja, violencia sexual e ideación suicida (Risser et al., 2005). Entre las MT colombianas el consumo de marihuana y alcohol muestra cifras más altas, un porcentaje un poco más bajo en el uso de basuco, pero mayores al compararlo con el consumo de esta sustancia en MT de República Dominicana que fue del 5,3\% (Budhwani et al., 2017). Además, los hallazgos son consistentes con los de la Encuesta de Consumo de SPA en Colombia, que muestra que la sustancia ilegal de mayor consumo es la marihuana, seguido de la cocaína, por otro lado, se reporta que solo el 1,2\% de las personas encuestadas había consumido basuco alguna vez en la vida (Keuroghlian, Reisner, White y Weiss, 2015; Ministerio de Justicia y del Derecho y Ministerio de Salud y Protección Social, 2014).

La relación entre alcohol o uso de drogas recreativas, comportamientos sexuales de riesgo y las ITS es bastante sólida y es conocida especialmente en investigaciones con HSH como "chemsex", resultados consistentes fueron encontrados en este estudio, en el cual el uso del basuco aumentaba la probabilidad de tener una ITS (Organización de los Estados Americanos, 2014; SIIS Centro de Documentación y Estudios, 2014). Según la literatura el consumo sustancias psicoactivas, incrementa los comportamientos sexuales de riesgo, como tener múltiples parejas o encuentros sexuales en una sola noche, intercambiar sexo por droga, la capacidad de negociar encuentros sexuales inseguros, o sufrir violencia sexual, la cual mostró en este estudio asociación estadística (Valencia et al., 2018). Si bien no se encontró asociación entre el basuco y la infección por VIH, como si ha sido reportado en otras investigaciones, un porcentaje importante de las MT que usan esta droga, resultaron seropositivas (Millar et al., 2018).

La salud mental y el consumo de drogas en MT se reporta como un problema importante de la población, sin embargo, la recolección sistemática de esta información es nula, ya que no existen encuestas sobre este tema, dirigidas específicamente a la población trans, no solo por las dificultades que acarrea el muestreo, sino también porque no se encuentran priorizadas en la agenda pública en salud. En Colombia son pocas las estrategias de prevención de consumo de SPA con enfoque diferencial y los programas se han centrado especialmente en escolares, adolescentes y habitantes de calle. Las MT han sido un grupo excluido de los programas sociales y en general se encuentran en entornos laborales y sociales problemáticos, en los cuales la venta y consumo de drogas es asequible, en especial el basuco por su bajo costo.

Por lo anterior se recomienda incentivar la creación de políticas y programas con un abordaje integral en salud, que tengan como uno de los ejes de acción la prevención del consumo de SPA en la población trans, teniendo en cuenta sus particularidades y características sociodemográficas y económicas.

$\mathrm{Al}$ ser una investigación transversal, no se logró establecer la secuencia temporal ni causalidad. En algunos casos las MT pueden olvidar detalles en algunas preguntas, al estar basados en los auto reportes; el RDS es considerado una muestra dependiente de la red social, la cual puede te- 
ner limitaciones al reportar las estimaciones poblacionales, afectando la generalización de los resultados obtenidos.

Este estudio tiene como fortalezas ser el primero realizado en MT colombianas bajo la metodología del RDS, además es el primero en reportar el consumo de basuco en esta población, lo que podría ayudar a implementar estrategias de prevención. Se recomienda realizar estudios de salud mental en población trans, que profundicen en los factores asociados al consumo, incluir a población trans joven, pues son pocos los datos en Colombia respecto a esta población y los inicios o patrones de consumo.

\section{Reconocimientos}

A todas las mujeres transgénero que aceptaron participar en el estudio, gracias a su valiosa colaboración e información. A las personas que participaron como "semillas" que por su sentido de pertenencia con el estudio fue posible acceder a la población en cada una de las ciudades del estudio.

La financiación para este estudio provino del Fondo Mundial de Lucha contra el Sida, La Tuberculosis y La Malaria, en el marco del Acuerdo de Subvención, N 216146 suscrito con La Empresa Nacional Promotora de Desarrollo Territorial -Enterritorio, quien, a su vez, subscribió un contrato con la Universidad CES en Medellín.

\section{Conflicto de intereses}

Las autoras del presente artículo declaran que no existe conflicto de intereses.

\section{Referencias}

Bazargan, M. y Galvan, F. (2012). Perceived discrimination and depression among low-income Latina male-to-female transgender women. BMC Public Health, 12, 663-670. doi:10.1186/1471-2458-12-663.

Berbesi-Fernández, D.Y., Segura-Cardona, Á.M., Montoya-Vélez, L. y Hernández-Rendón, M. (2016). Consumo de basuco en usuarios de drogas inyectables en Colombia. Revista Cubana Salud Pública, 42, 276-283.

Berbesi-Fernández, D.Y., Segura-Cardona, Á., Martínez-Rocha, A., Molina-Estrada, A., Ramos-Jaraba, S. M. y Bedoya-Mejía, S. (Eds.) (2019). Vulnerabilidad al VIH y prevalencia de VIH en mujeres transgénero en tres ciudades de Colombia: Bogotá, Medellín y Santiago de Cali. Medellín: Universidad CES Editorial.

Budhwani, H., Hearld, K. R., Milner, A. N., McGlaughlin, E., Charow, R., Rodriguez-Lauzurique, R. M.,... Paulino-Ramirez, R. (2017). Transgender women's drug use in the Dominican Republic. Transgender Health, 2, 188194. doi:10.1089/trgh.2017.0032.
Carballo-Diéguez, A., Balan, I., Marone, R., Pando, M. A., Dolezal, C., Barreda, V.,... Ávila, M. M. (2011). Use of respondent driven sampling (RDS) generates a very diverse sample of men who have sex with men (MSM) in Buenos Aires, Argentina. PLoS One, 6. doi:10.1371/journal.pone.0027447.

Comisión Interamericana de Derechos Humanos. (2015). Violencia contra las personas Lesbianas, Gay, Bisexuales, Trans e Intersexuales en América [OEA/Ser.L/V/ II. Rev.2.Doc. 36]. Recuperado de http://www.oas.org/ es/cidh/informes/pdfs/ViolenciaPersonasLGBTI.pdf.

Dávila, L., Solórzano, E., Premoli de Percoco, G., Quiñones, B. y Petrosino, P. (2001). El consumo de basuco como agente causal de alteraciones en la encía. Revista Cubana Estomatología, 38, 137-144.

Ecker, J., Aubry, T. y Sylvestre, J. (2019) . A review of the literature on LGBTQ adults who experience homelessness. Journal of Homosexuality, 66, 297-323. doi:10.1080/00918 369.2017.1413277.

Gómez-Gil, E., Simulionyte, E., Balcells-Oliveró, M., Valdés, M., Salamero, M., Guillamón, A. y Esteva, I. (2019). Patrones de consumo de alcohol, tabaco y drogas ilegales en personas transexuales. Adicciones, 31, 189-195. doi:10.20882/adicciones.945.

Family Health International. (2000). Behavioral Surveillance Surveys. Arlington, VA: Family Health International.

Isaza, C., Suárez, P., Henao, J. y González, M. (2010). Características demográficas y de consumo en pacientes adictos a heroína o derivados de la coca. Investigaciones Andina, 12, 24-34.

Keuroghlian, A. S., Reisner, S. L., White, J. M. y Weiss, R. D. (2015). Substance use and treatment of substance use disorders in a community sample of transgender adults. Drug and Alcohol Dependence, 152, 139-146. doi:10.1016/j. drugalcdep.2015.04.008.

Larrea-Torrelio, G. A. (2007). Historia y producción de drogas en el siglo XX, hoja de coca, cocaina y fármacos. Tesis Doctoral en Economía. La Paz: Repositorio Institucional Universidad Mayor de San Andrés.

Millar, B. M., English, D., Moody, R. L., Rendina, H. J., Cain, D., Antebi-Gruszka, N.,... Parsons, J. T. (2018). Day-level associations between substance use and HIV risk behavior among a diverse sample of transgender women. Transgender Health, 3, 210-219. doi:10.1089/ trgh.2018.0032.

Ministerio de Justicia y del Derecho y Ministerio de Salud y Protección Social. (2014). Estudio nacional de consumo de sustancias psicoactivas - 2013. Recuperado de https:// cutt.ly/7bXMhHo.

Ministerio de Salud y Protección Social. (2015). Encuesta nacional de salud mental 2015. Recuperado de https:// cutt.ly/IbXCQfI. 
Observatorio Uruguayo de Drogas. (2014). Pasta base de cocaina en Uruguay. Recuperado de https://cutt.ly/ ibX097S.

Organización de los Estados Americanos. (2014). Consumo de pasta base de cocaína en América del Sur: Revisión de los aspectos epidemiológicos y médico-toxicológicos. Recuperado de https://cutt.ly/ObX05My.

Organización Panamericana de la Salud. (2011). Por la salud de las personas trans. Elementos para el desarrollo de la atención integral de personas trans y sus comunidades en Latinoamérica y el Caribe. Recuperado de https:/ / pdf.usaid. gov/pdf_docs/PA00JQ81.pdf.

Radi, B. y Sardá-Chandiraman, A. (2016). Travesticidio / transfemicidio: Coordenadas para pensar los crímenes de travestis y mujeres trans en Argentina. [Boletín del Observatorio de Género, 9]. Recuperado de https:// www.aacademica.org/blas.radi/14.pdf?view.

Reback, C. J. y Fletcher, J. B. (2014). HIV prevalence, substance use, and sexual risk behaviors among transgender women recruited through outreach. AIDS and Behavior, 18, 1359-1367. doi:10.1007/s10461-013-0657-z.

Risser, J. M. H., Shelton, A., McCurdy, S., Atkinson, J., Padgett, P., Useche, B.,...Williams, M. (2005). Sex, drugs, violence, and HIV status among male-to-female transgender persons in Houston, Texas. International Journal of Transgenderism, 8, 67-74. doi:10.1300/J485v08n02_07.

Santos, G. M., Rapues, J., Wilson, E. C., Macias, O., Packer, T., Colfax, G. y Raymond, H. F. (2014). Alcohol and substance use among transgender women in San Francisco: Prevalence and association with human immunodeficiency virus infection. Drug and Alcohol Review, 33, 287-295. doi:10.1111/dar.12116.

Sandoval-Rebollo, E. M., Domínguez-Cornejo, M. M. y Rosales-Galarza, E. R. (2019). La situación de acceso a derechos de las personas trans en México: Problemáticas y propuestas. México: Embajada de los Estados Unidos en México. Recuperado de https://almascautivasorg. files.wordpress.com/2019/02/la-situacic3b3n-de-acceso-a-derechos-de-las-personas-trans-en-mc3a9xico-investigacic3b3n-completa.pdf.

Scheim, A. I., Bauer, G. R. y Shokoohi, M. (2017). Drug use among transgender people in Ontario, Canada: Disparities and associations with social exclusion. Addictive Behaviors, 72, 151-158. doi:10.1016/j.addbeh.2017.03.022.

Scher, A. (2016). Estigma y discriminación hacia hombres que tienen sexo con otros hombres (HSH) y mujeres trans: El impacto en la vulnerabilidad y riesgo frente al VIH/SIDA. [Independent Study Project (ISP) Collection. 2461]. Recuperado de https://digitalcollections. sit.edu/isp_collection/2461.

SIIS Centro de Documentación y Estudios. (2014). Desigualdades socioeconómicas, consumo de drogas y territorio. Recuperado de https://cutt.ly/RbXM1nC.
SInViolencia LGBT. (2019). El prejuicio no conoce fronteras. Homicidios de lesbianas, gay, bisexuales, trans e intersex en países de América Latina y el Caribe 2014-2019. Recuperado de https://cutt.ly/9bXGAVZ.

Téllez-Mosquera, J. y Cote-Menéndez, M. (2005). Efectos toxicológicos y neuropsiquiátricos producidos por consumo de cocaína. Revista de la Facultad de Medicina, 53, $10-26$

Valencia, J., Gutiérrez, J., Troya, J., González-Baeza, A., Dolengevich, H., Cuevas, G. y Ryan, P. (2018). Consumo de drogas recreativas y sexualizadas en varones seronegativos: Datos desde un screening comunitario de VIH. Revista Multidisciplinar del SIDA, 6, 7-19.

Yarns, B. C., Abrams, J. M., Meeks, T. W. y Sewell, D. D. (2016). The mental health of older LGBT adults. Current Psychiatry Reports, 18, 1-11. doi:10.1007/s11920-0160697-y. 
